\title{
How heat stress drives the expression of LTR retrotransposons in the flatworm model organism Macrostomum lignano
}

\author{
Ustyantsev K. ${ }^{1 *}$, Wudarski J. ${ }^{2}$, Vavilova V. ${ }^{1}$, Berezikov E. ${ }^{1,2}$ \\ ${ }^{1}$ Institute of Cytology and Genetics, SB RAS, Novosibirsk, Russia \\ ${ }^{2}$ European Research Institute for the Biology of Ageing, UMCG, Groningen, The Netherlands \\ *e-mail: ustyantsev@bionet.nsc.ru
}

Key words: heat shock, LTR retrotransposons, transgenesis

Motivation and Aim: There is a growing body of evidence suggesting that some transposable elements are activated under stress conditions. However, the exact molecular mechanism underlying the activation is rarely known. Recently, we have found that two groups of Ty3/Gypsy LTR retrotransposons (LTR-RTs) are highly expressed under heat shock $\left(37^{\circ} \mathrm{C}\right)$ in the genome of a free-living flatworm Macrostomum lignano, a model species to study stem cell biology, regeneration and ageing [1]. Here, we present new evidence for the activation mechanism of these retrotransposons, confirming our previous hypothesis with the new experimental data.

Methods and Algorithms: We utilized the transgenesis technology [2] to investigate whether the long terminal repeat (LTR) sequence of the LTR-RTs alone can induce expression of the reporter fluorescent protein in $M$. lignano after heat shock, and used the promoter of the heat shock protein gene $(H s p) 20$ as a positive control [3].

Results: Three new M. lignano transgenic lines were produced. The heat shock treatment resulted in the expression of the fluorescent reporter controlled both by the LTRs and the Hsp 20 promoters of the transgenic worms. The LTR-driven expression was mostly absent from gonads, while it was ubiquitous in the Hsp20-line.

Conclusion: The functional elements of the heat stress activation of the M. lignano LTRRTs are located within their LTRs and regulated by the same mechanism as that for Hsp genes.

Acknowledgements: The study was supported by the RFBR grant No. 18-34-00288.

\section{References}

1. Vavilova V. et al. Heat shock response elements are present in the promoters of the heat stress activated LTR retrotransposons in the free-living regenerative flatworm Macrostomum lignano. Systems Biology and Bioinformatics (SBB-2018). The Tenth International Young Scientists School. - Novosibirsk, $2018 ; 46$.

2. Wudarski J. et al. Efficient transgenesis and annotated genome sequence of the regenerative flatworm model Macrostomum lignano. Nat Commun. 2017;8:2120.

3. Wudarski J. et al. Influence of temperature on the development, reproduction and regeneration in the flatworm model organism Macrostomum lignano. Zoological Letters. 2019;5:7. 